\title{
Teaching color and color science: the experience of an international master course
}

\author{
Maurizio Rossi; Politecnico of Milano; Milano, Italy \\ Alice Plutino; Università degli Studi di Milano; Milano, Italy \\ Andrea Siniscalco; Politecnico of Milano; Milano, Italy \\ Alessandro Rizzi; Università degli Studi di Milano; Milano, Italy
}

\begin{abstract}
There are not many international courses that teach color science applied to non-technical fields. Here, we want to present the experience from a master course organized by Gruppo del ColoreAssociazione Italiana Colore and Politecnico of Milano: the Master in Color Design \& Technology. It has the aim of training students in the use of color mainly for design purposes, and color science and colorimetry have a big role in the program. The Master is organized in three main phases: Fundamentals, Project Works and Internship. The first part is a series of lectures and frontal lessons that gives to the students the theorical and technical bases to be used in project works practical applications of and internship. In fact, in the second and third parts students are asked to design and develop different applicative design projects. After the first part of Fundamentals training, students will be able to manage disciplines such as colorimetry, visual perception, physics, chemistry, optics and psychology, to build up their future professional career in different fields.
\end{abstract}

\section{Introduction}

The use and meaning of color is usually taught since the kindergarten, because of its fascinating and captivating nature. Colors are used to draw, represent and communicate the surrounding environment, and the multidisciplinary nature of color can be the base of many investigations.

Color is often described as a property of the matter, but it does not exist without a human being perceiving it. On the other side, it is used to describe and distinguish different objects, but it is extremely depending on the context.

The master aims at presenting the multidisciplinary nature of color and the many ways and standards to communicate, use, measure and represent color. The course is unique in the world and during the last three editions, students from all over the world have attended it. We had students from: Australia, Brazil, Colombia, India, Italy, Japan, Lebanon, Spain, Portugal, Russia and many other countries. The multicultural environment in which the students are involved enriches them and permit the development of a positive discussion about color culture, naming and uses across different nations. Furthermore, the master is open to students with diverse backgrounds, the majority of them come from architecture, interior design and product design, but we have had also students specialized in economics, management, cultural heritage or visual arts. All of them are looking for a specialization in color applications and to complete each own knowledge on color, especially during the project works, during where they are expected to work in groups.

The master in Color Design and Technology has three main phases. The first one is theorical and aims at developing the students' technical competences in color physics, measuring, digital management, reproduction and results comparisons, starting from subjects like physics, optics, colorimetry, chemistry, psychology and perception [1]. In the second and third phase the students are asked to apply the learned competences through group projects and an internship.

This master is held by Politecnico of Milano in collaboration with the Gruppo del colore - Associazione Italiana Colore, the Italian color association, and has the main objective of educating future professionals able to succeed in all the technological issues concerning colour management. Due to the multidisciplinary nature of colour applications, during the course, students deal with different field of application like industrial product design, interior design, communication, fashion design, urban spaces and many others. During the master, colour won't be tought as simple attribute of an object, but as technical means of expression, at the base of perception and interaction with reality.

\section{The educational approach}

To teach color there are many different approaches, strictly linked with the final application and with the field of study. For example, in art academies the color is a varnish, or a pigment that students learn to manage and mix to create the perfect tone to express a concept; in a different field, like physics, color is taught as property of the matter dependent on to the incident and reflected spectral radiation; alternatively, in psychology color is an attribute of the matter interpreted by the human brain that constructs meaning based on its experience.

The field of science that aims at measuring colors is colorimetry and it is at the base of the major part of the color teaching approaches. It is a powerful tool for color description, communication and standardization, but on one side many students often tend to address to it just as a theorical topic, without really understanding all its practical applications, while on the opposite side other students identify colorimetry as the only way to approach color, forgetting in this way the strong limiting assumption of this field of research: the aperture mode assumption.

The master in Color Design and Technology aims at bridging the gap between colorimetry and different color application projects in the domain of design. Through the master we do not want to educate experts in colorimetry, but to train creative professionals that could apply colorimetric notions in their working life. This aim is the challenge of the master: our students are not just asked to learn the basis of colorimetry and color systems, but also to apply, manage and understand them in many different fields, through the Project Works applications and during the internships.

In order to achieve this goal, the master Fundamentals are organized in theorical modules, but lessons are always combined with color applications instances and empowerments made by professional, manufactures and studios. 
Due to the paramount importance that new technologies have in the work of today designers, one important novelty of the third edition of the master, is the improvement of the lessons of the Digital Color module. Here, in fact, are explained the modern digital color management techniques using last generation calibrated monitors and projectors, experimenting and controlling color changes across different devices gamuts. Attention is paid also to devices calibration and characterization, to increase the students' awareness in the limitations and potentials of professional devices.

As a positive result of the first two Master editions is that the major part of the students actually works in the field of color as expert in companies or as freelancers. Furthermore, it has also happened that some students making the internship in a company, which specific business was not directly related with color, ended up making suggestions that improved the company business workflow.

\section{The structure}

\section{Fundamentals}

The Fundamentals cover color culture, history and scientific basis through classroom lessons, together with seminaries made by professionals. They consist in about 200 hours of lessons and 32 hours dedicated to professional empowerment with companies working in the field of color.

The first module of the Fundamentals presents the history of color discoveries [2] and the aspects related to visual perception. Many students already know and use the color wheels, but few of them remember that one of the first men to present one was Isaac Newton, and everyone knows how to distinguish a primary from a secondary color, but it is important to reconduct it to the studies of Goethe. So, starting from the history of color theories it is possible to understand all the multidisciplinary nature of color, and to study its physics, psychology and sociology.

In the first module we focus also on color perception, and the mechanism that affects color and vision are examined, to enable the students to recognize, anticipate and design them [3]. We focus in particularly on phenomena like simultaneous contrast, assimilation, color in context because, in our experience, we found that their study could really improve the understanding of the main color management issues [1].

In this first module, the students are also introduced to the subject of color naming, starting from the theories of Berlin and Kay [4]. In this context, during this module, students are often asked to give us their opinion and feedback about the use of colors and color naming in their nation, culture and language, enriching the didactics.

The second module presents colorimetry [5] and color systems, explaining how we can represent numerically a color and how the color vision is simplified and standardized.

In this module we start from teaching how to measure a color, defining the illuminant and acquiring the reflectance of a surface. The lessons concentrate on giving to the students the basis of colorimetry, photometry and radiometry, as well as an overview of the human visual system [6]. After this module students are capable of choosing the right illuminant for a specific purpose due to its technical characteristics and know how to represent the same color in different color spaces. We support the lectures with practical trainings using spectrophotometers, colorimeters and spot meters.

The third module of the Fundamentals is Digital Color. In this module students face with color representation through different devices. We dedicate to the digital color an entire module, because, today, it is fundamental for a color manager and color consultant to be able to control color physically and digitally.

This module gives to the students the basic knowledge and instruments to manage, view and reproduce a color through different media. At first, notions of device calibrations, characterization and gamut are given to the students [7]. Furthermore, this module provides a comprehensive knowledge of the processing chain and digital reproduction, but also of the main problems and issues linked with color digitization [8], [9].

As in the previous modules, thanks to the collaboration with different professionals, students can make different tests and applications of color correction and management for advertisement or artistic purpose (see Figure 1).

All along the fundamentals are spread empowerment lessons and practical cases by professionals. We schedule the fundamentals in the way that for each issue and subject addressed, a practical application is presented from experts in many different fields like e.g. jewelry, photography, advertisement, film color grading, etc.

As another example, during the last edition we combined lectures about the physics of color with cultural heritage applications, so the students can see how the reflectance spectroscopy can be used to make identification of pigments and recognize false paintings.

In general, we found that, the union of empowerments with color theory modules are very useful to support the students' process of personal growth by developing the ability of form relationships and work successfully in a variety of fields.
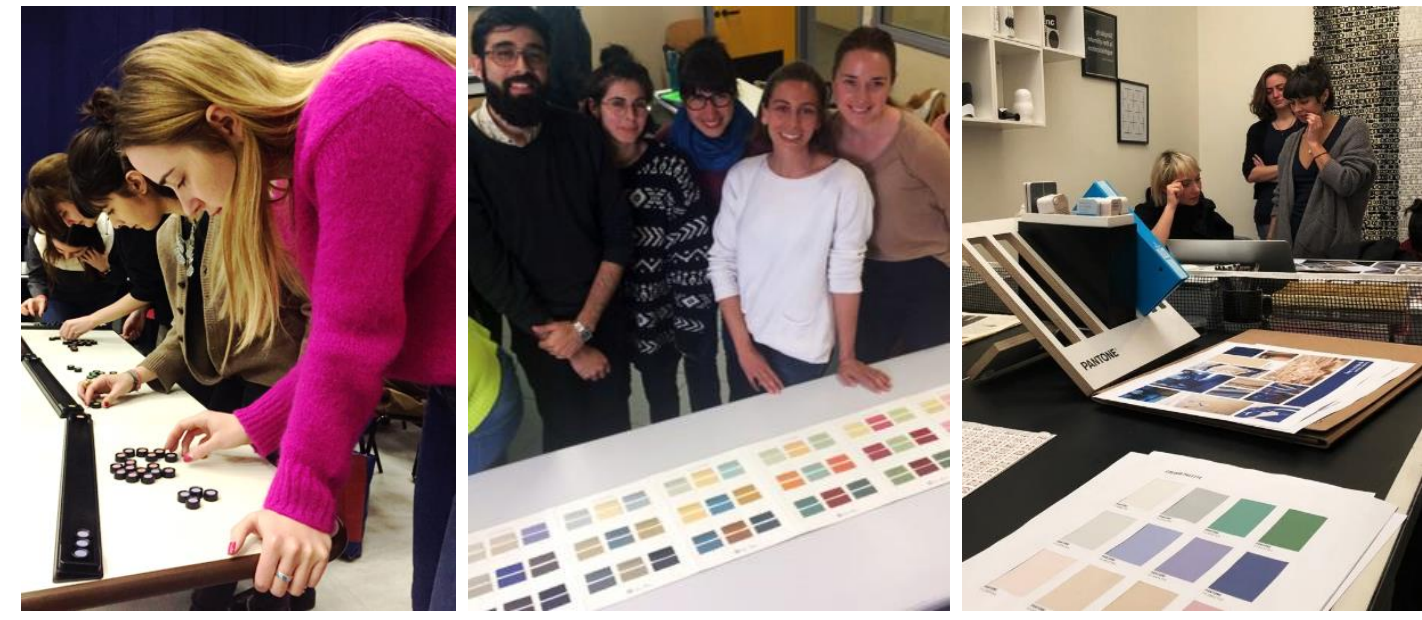

Figure 1 Pictures taken during the Fundamental And the ProjeCt Works. 


\section{Project Works}

The second part of the master is dedicated to the Project Works. Like the Fundamentals, this part consists in about 200 hours divided into five projects, to make the students apply what they learned during the first part.

In this phase students are asked to work in groups. Thanks to the variety of skills and backgrounds of the students, the environments of the projects has always been dynamic and enriching (see Figure 1).

The module of the Project Works refers to typical color designs scenarios and focus on five main topics: Communication, Fashion Design, Industrial Product and Urban Space.

The first Project Work focuses on the use of color in communication. Students study the importance of the brand identity for a company and all the ways in which it can be communicated through colors in the brand, packaging, name, lettering and so on. The color is seen as one of the key components of the brand identity and it is built on the evocative and persuasive aspects of the chromatic language.

The second Project Work is dedicated to the study of color texturing in fashion with the aim of designing products with color variations to reach culturally different markets and propose a guided personal choice to customers. Through this project, students create a textile color book, build a mood-board, experiment how to construct a color palette and how to combine the textiles and fabrics in harmonious way.

The third Project Work focuses on the use of color for interior design and is dedicated to the analysis of the application of a chromatic design in indoor environment, like e.g. commercial spaces. Starting from practical examples of different applications of retail design, students have to propose new type of commercial spaces, permanent or temporary, where color is the key component of the design experience.

The Industrial Product Project Work aims at developing a method to design an industrial product through the choices of Color/Material/Finishes composing it. Before the creation of a new product, are shown to the students different color samples used in the market and many prototypes ready for the commercial launch, and after this part, students start to design colors, materials and at the end the final product.

The last Project Work concerns the use of color for Urban Space. Here, the aspects related to the interaction between the human being and the urban environment are investigated. In this project, to the students are provided case studies and procedural tools to correlate the color perception in the urban space to build visual identities.

\section{Conclusions}

Color is a fascinating theme that involves many disciplines like physics, chemistry, optics, psychology, anthropology and sociology, not just colorimetry and computer science. It is related with the visual perception and has an impact on the cognitive and emotional nature of the human being. We are surrounded by colors, at home, in the cities, at the supermarket. Despite this, the lack of universal color concepts, rules and standards makes forming related professionals complex, but very necessary.

In this paper we showed the structure of the master in Color Design and Technology, held in Italy and entering its fourth edition. It is one of the first masters in the world, with the aim of forming professionals with both technical and design skills.
During the previous master editions, we have seen an increasing satisfaction of the companies that collaborate with us for lessons, empowerment and student internship, and this increase consequently student interest.

The master is held in English and the students come from all around the world, but teachers and companies are mainly based in Italy. One of the goal of this paper is also fostering possible collaboration at an international level.

\section{References}

[1] M. Rossi, «Colour Education: a Basic Methodology and a Framework of Experiments for Colour and Lighting Design Teaching,» AIC2016 Interim Meeting of the International Colour Association (AIC) Color in Urban Life: Images, Objects and Spaces, 2016.

[2] P. Ball, Bright Earth: Art and the Invention of Color, University of Chicago Press, 2003.

[3] D. Hoffman, «The interface theory of perception: Natural selection drives true perception to swift extinction,» Object categorization: Computer and human vision perspectives, Cambridge, UK 2009.

[4] B. Berlin e P. Kay, «Basic Color Terms: Their Universality and Evolution,» University of California Press, 1969.

[5] C. Oleari e G. Simone, Standard Colorimetry: Definitions, Algorithms and Software (SDC-Society of Dyers and Colourists), Wiley, 2015.

[6] G. Wyszecki e W. Stiles, Color Science: Concepts and Methods, Quantitative Data and Formulae, Wiley, 2000, pp. "Color Science: Concepts and Methods, Quantitative Data and Formulae", 2nd ed., Wiley. 2000.

[7] R. Ramanath, W. Snyder, Y. Yoo e M. Drew, Color image processing pipeline, IEEE Signal Processing Magazine 22(1) 34-43, 2005.

[8] J. McCann e A. Rizzi, The Art and Science of HDR Imaging, Wiley, 2011.

[9] J. McCann, V. Vonikakis e A. Rizzi, HDR Scene Capture and Appearance, SPIE Spotlight Series, Volume: SL35, 2017.

[10] S. Braddock e M. O'Mahony, Techno Textiles: Revolutionary Fabrics for Fashion and Design, Thames \& Hudson, 1999.

[11] A. Fletcher, Beware wet paint, Phaidon, 2004.

[12] C. Lefteri, Plastic, materials for inspirational design, Rotovision, 2001.

[13] M. Webster, The Merriam-Webster Dictionary, Merriam Webster Mass Market, 2016. 


\section{Author Biography}

Maurizio Rossi, MSc, PhD. Full professor of Lighting Design at

Politecnico di Milano where he is the scientific manager of the Lab. Luce, and the director of the master in Color Design and Technology and the

Master in Lighting Design \& Technology. From 2012 to 2018 he was the President of the Associazione Italiana Colore. He is the editor-in-chief of the Color Culture and Science Journal. Since 2018 is member of the Executive Committee of AIC (International Color Association).

Alice Plutino is a PhD student in Computer science at the university of Milan. She obtained her master's degree in Conservation Science for Cultural Heritage at the same university. Now, she is focusing on color correction, image enchantment and image quality assessments. She is author of different papers concerning innovative methods for film restoration and digital restoration assessments.

Andrea Siniscalco, MSc in Industrial design in 2002. PhD in design and methods of Product development in 2007. Vice director and teacher in the master in Lighting Design \& LED Technology of Politecnico di Milano. Adjunct professor in Lighting theory at the School of Design. Vice president of GdC - Associazione Italiana Colore. Fields of interest are lighting design, visual perception and color.

Alessandro Rizzi is Full Professor at the Department of Computer Science at the University of Milan. From 1990 his research has been in the field of colour, digital imaging and vision. He is the head of the MIPS Lab at the Department of Computer Science and was one of the founders of the Italian Colour Group, Secretary of CIE Division 8, IS\&T Fellow and Vice President. In 2015 he received the Davies medal from the Royal Photographic Society. He is co-chair of the IS\&T Conference "Color Imaging: Displaying, Processing, Hardcopy and Applications”, a member of several programme committees of conferences related to colour and digital imaging, and author of about 300 scientific works. 